\title{
Uma métrica para avaliação da ocupação docente em instituições de ensino superior
}

\author{
A metric for teaching workload \\ evaluation in higher education institutions
}

\author{
Atilio Minotto Neto ${ }^{1}$ \\ Leandro Luis Corso ${ }^{2}$ \\ Guilherme Holsbach Costa ${ }^{3}$
}

Resumo: A gestão da ocupação docente em instituições de ensino superior é um problema que afeta universidades públicas e, principalmente, as privadas. Uma distribuição justa de tarefas aos docentes repercute diretamente na qualidade do ensino, na produção de conhecimento, na sustentabilidade institucional e, principalmente, na saúde dos docentes. Neste trabalho é proposto um método para avaliação da composição da carga de trabalho com base em uma ferramenta chamada Processo Analítico Hierárquico, visando a equidade em termos de carga de trabalho (o que não significa o mesmo que equidade das cargas-horárias nas diferentes atividades docentes). Como principal contribuição, é proposta uma métrica, chamada Coeficiente de Carga de Trabalho, que pode ser facilmente adaptada a realidade de cada instituição. Apesar das bases matemáticas, quando implementada computacionalmente, a utilização dessa métrica passa a ser simples e acessível a gestores de qualquer formação.

Palavras-chave: Carga de trabalho. Ocupação docente. Processo analítico hierárquico. 
Abstract: The management of teaching occupation in higher education institutions is a problem that affects public and, mainly, private universities. A fair distribution of tasks to teachers directly affects the quality of teaching, the production of knowledge, institutional sustainability and, mainly, the health of teachers. This work proposes a method for evaluating the composition of the workload based on a tool called the Hierarchical Analytic Process, aiming at equity in terms of workload (which does not mean the same as equity in the amount of hours dedicated to different activities). As the main contribution, a metric, called Workload Coefficient, is proposed, which can be easily adapted to the reality of each institution. Despite the mathematical bases, when implemented computationally, the use of this metric becomes simple and accessible to managers in any area of knowledge.

Keywords: Workload. Teaching occupation. Hierarchical analytic process.

\footnotetext{
${ }^{1}$ Universidade de Caxias do Sul | Programa de Pós-graduação em Engenharia Mecânica | Caxias do Sul |RS | Brasil. Contato: amneto2@ucs.br. ORCID: https://orcid.org/0000-0002-8802-0318
}

${ }^{2}$ Universidade de Caxias do Sul | Programa de Pós-graduação em Engenharia Mecânica | Caxias do Sul | RS | Brasil. Contato: 1lcorso@ucs.br. ORCID: https://orcid.org/0000-0001-9962-9578

${ }^{3}$ Universidade de Caxias do Sul | Programa de Pós-graduação em Engenharia Mecânica | Caxias do Sul | RS | Brasil. Contato: ghcosta@ucs.br. ORCID: https://orcid.org/0000-0003-1742-5331

- Recebido em: 14 de junho de 2020

- Aprovado em: 11 de novembro de 2020

DOI: http://dx.doi.org/10.1590/S1414-40772021000200005

Este é um artigo publicado em acesso aberto sob uma licença Creative Commons https://creativecommons.org/licenses/by-nc/4.0/ 


\section{Introdução}

Entre os anos de 2006 e 2018 houve um aumento de cerca de 18\% no número de docentes de ensino superior no Brasil (INEP, 2018). Neste mesmo período, o censo de 2018 aponta um aumento de mais de $82 \%$ no número de DTI's frente a uma redução de mais de $50 \%$ no número de horistas (INEP, 2018). Nas instituições de ensino superior privadas, o crescimento no número de professores é inexpressivo, de 2016 a 2018 (apenas 1\%), mas o número de DTI's e DTP's cresceu $80 \%$, frente a uma diminuição também de mais de $50 \%$ no número de horistas. Essa migração de DH's para DTP's e DTI's nas instituições privadas tem impacto direto na sustentabilidade, o que ressalta a necessidade de um planejamento que busque o bom aproveitamento dos recursos humanos. Note-se que esses enquadramentos diferem entre si pela proporção de cada atribuição do docente, isto é, a carga horária total é dividida entre um conjunto de possíveis atividades para cada regime de trabalho, cada uma delas, nas instituições não estatais, com um retorno financeiro (direto) distinto.

No Brasil o Ministério da Educação (MEC) regulamenta os regimes de trabalho da seguinte forma (BRASIL, 2002):

- docentes em tempo integral - aqueles contratados com 40 horas semanais em uma mesma Instituição de Ensino Superior (IES), das quais pelo menos 20 horas são destinadas a pesquisa, extensão, gestão ou outras atividades além do ensino;

- docentes em tempo parcial - aqueles contratados com 12 horas semanais ou mais em uma mesma instituição, nas quais pelo menos $25 \%$ do tempo é reservado para estudos, planejamento, avaliação e orientação de alunos;

- docentes horistas - aqueles contratados exclusivamente para atividades de ensino, independentemente da carga horária, ou que não se enquadrem nas outras categorias.

As políticas que regem a ocupação de cada docente variam de instituição para instituição e, de forma geral, consideram poucas variáveis e que nem sempre representam de forma fidedigna as demandas enfrentadas por diferentes professores de um mesmo enquadramento, gerando disparidades em suas cargas de trabalho e percepções de injustiça. Por exemplo, ao pautar a ocupação docente por uma carga-horária mínima em atividades de ensino, à luz da regulamentação do MEC, não se considera se essa nessa carga-horária o professor ministra várias turmas de uma mesma disciplina ou de várias disciplinas distintas, o que, neste último caso, seguramente demanda mais trabalho na preparação das aulas. De forma similar, não se considera se as turmas possuem muitos ou poucos alunos, o que além de demandar um diferente volume de trabalho impacta diretamente na sustentabilidade. Apesar dos diferentes modelos gerenciais, tanto instituições públicas quanto privadas precisam garantir a continuidade e a 
qualidade da prestação de serviços e tendem a se preocupar com a justiça na ocupação de seus docentes, tornando esse um problema comum às IES's de todas as naturezas.

De forma direta, as atividades de ensino são por via de regra as mais rentáveis para uma IES de direito privado, fazendo com que o planejamento da carga horária - compreendendo todas as dimensões do trabalho acadêmico — desempenhe um papel importante no alinhamento das metas institucionais com as metas individuais (BURGESS, 1996; BARRETT; BARRETT, 2007). Viana et al. (2019) aprofundam uma discussão sobre a gestão do trabalho docente no Brasil após uma reestruturação da legislação na década de 90, considerando aspectos como estrutura, organização e gestão político-institucional das universidades brasileiras.

Um sistema de gestão da carga horária deve ser verdadeiramente preocupado em evitar o estresse dos profissionais acadêmicos (BURGESS, 1996). Indicadores administrativos errôneos tendem a associar uma maior carga de trabalho a uma maior produtividade, enquanto, do ponto de vista dos profissionais, sobrecarga de trabalho é associada com estresse, fadiga, menor satisfação e produtividade reduzida. A adequada alocação de carga horária de docentes de uma IES, por envolver diversos fatores, requer uma cuidadosa análise sobre a influência desses critérios em sua composição, de forma que uma ferramenta matemática de apoio pode ser de grande valia ao gestor. O Processo Analítico Hierárquico (AHP - Analytic Hierarchy Process) é uma ferramenta utilizada no auxílio de tomada de decisões baseadas em diversos critérios. Neste trabalho é proposto um método para avaliação e controle da ocupação docente em uma IES com base no AHP. O método proposto visa a equidade na carga de trabalho dos docentes, o que não significa buscar um equilíbrio na carga-horária dedicada às diferentes atividades docentes.

\section{Processo Analítico Hierárquico}

O AHP propõe a estruturação de um problema em uma hierarquia composta pelo objetivo a ser alcançado, pelos critérios a serem analisados e pelas alternativas disponíveis, conforme ilustrado na Figura 1. Em sua estrutura básica, a hierarquia proposta no AHP define que $M$ características relevantes (chamadas aqui de critérios) contribuem com diferentes pesos na escolha de uma das $N$ alternativas disponíveis de modo a alcançar determinado objetivo. Isto se baseia na formulação de uma matriz de comparação cujos elementos são o resultado da comparação dos critérios entre si, com base em uma escala de importância (ver Tabela 1) — o que é conhecido como comparação de pares (BRUNELLI, 2014). 


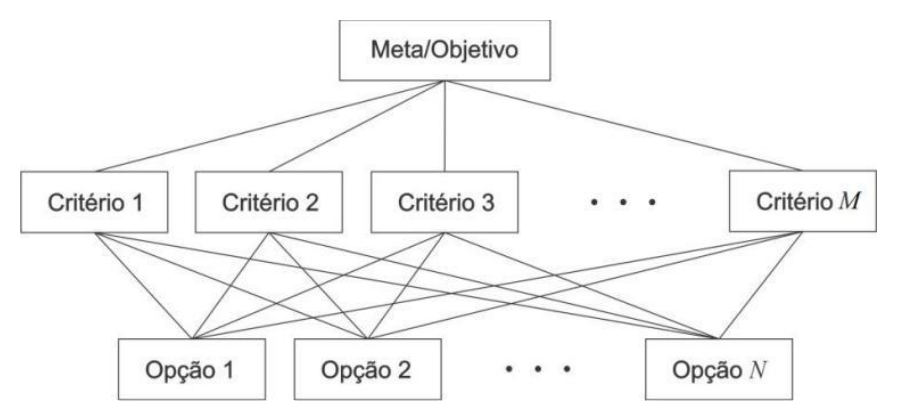

Fonte: Adaptado de Mu e Pereyra-Rojas (2017)

Tabela 1 - Escala fundamental AHP

\begin{tabular}{|c|c|c|}
\hline $\begin{array}{l}\text { Intensidade de } \\
\text { importância em } \\
\text { escala absoluta }\end{array}$ & Definição & Explicação \\
\hline 1 & Igual importância & Duas atividades contribuem igualmente para o objetivo \\
\hline 3 & $\begin{array}{c}\text { Importância moderada de uma sobre } \\
\text { a outra }\end{array}$ & $\begin{array}{l}\text { Uma atividade contribui mais para o objetivo do que a } \\
\text { outra }\end{array}$ \\
\hline 5 & Essencial ou grande importância & $\begin{array}{l}\text { Uma atividade contribui consideravelmente mais para o } \\
\text { objetivo do que a outra }\end{array}$ \\
\hline 7 & Importância muito grande & $\begin{array}{l}\text { Uma atividade é fortemente favorecida e sua dominância } \\
\text { é demonstrada na prática }\end{array}$ \\
\hline 9 & Extrema importância & $\begin{array}{l}\text { A evidência que favorece uma atividade sobre a outra é } \\
\text { da mais alta ordem de afirmação possível }\end{array}$ \\
\hline $2,4,6,8$ & $\begin{array}{c}\text { Valores intermediários entre } \\
\text { julgamentos adjacentes }\end{array}$ & $\begin{array}{l}\text { Quando se necessita de maior precisão na análise de } \\
\text { julgamentos }\end{array}$ \\
\hline
\end{tabular}

Fonte: Adaptado de Saaty (1990)

A comparação de pares seguindo a escala absoluta proposta por Saaty (1990) lida com uma avaliação empírica das características envolvidas e requer uma análise cautelosa. Isso se evidencia pelo fato de que a definição da importância de cada critério isoladamente (ou peso do $m$-ésimo critério, denotado por $\omega_{m}$ ) pode se tornar bastante complicada na prática.

Como alternativa, define-se então as importâncias relativas, denotadas por $a_{i j}$, que representam a importância do critério $i$ sobre o critério $j$ conforme a escala absoluta mostrada na Tabela 1 . Os coeficientes $a_{i j}$ podem ser arranjados em uma matriz, denominada matriz de comparação de pares, ou simplesmente matriz de comparação, na forma 


$$
\begin{aligned}
& \begin{array}{llll}
A_{1} & A_{2} & \cdots & A_{M}
\end{array} \\
& \left.\mathbf{A}=\left(a_{i j}\right)_{M \times M}=\underset{A_{1}}{A_{2}} \begin{array}{cccc}
a_{11} & a_{12} & \ldots & a_{1 M} \\
a_{21} & a_{22} & \ldots & a_{2 M} \\
\vdots & \vdots & \ddots & \vdots \\
A_{M} & a_{M 2} & \ldots & a_{M M}
\end{array}\right]
\end{aligned}
$$

em que $A_{m}$ é o $m$-ésimo critério considerado. Por conseguinte, o problema dos pesos de cada critério pode ser interpretado como

$$
a_{i j} \approx \frac{\omega_{i}}{\omega_{j}} \quad, \quad i, j=1,2, \ldots, M
$$

em que $\omega_{i}$ e $\omega_{j}$ são os pesos do $i$-ésimo e $j$-ésimo critérios respectivamente. Nota-se que ao realizar a comparação empírica de pares entre as características, apenas os coeficientes $a_{i j}$ são conhecidos. Segundo Saaty (1990), isso acontece pois em um ambiente de tomada de decisão, os valores de $\omega_{i} / \omega_{j}$ não são obtidos de forma precisa, apenas suas estimativas.

O problema agora recai na determinação dos pesos $\omega_{m}$ a partir da matriz $\mathbf{A}$. Para esse fim, pode-se partir de um sistema de equações lineares $\mathbf{A w}=\mathbf{b}$, em que $\mathbf{w}=$ $\left[\begin{array}{llll}\omega_{1} & \omega_{2} & \cdots & \omega_{M}\end{array}\right]^{\mathrm{T}}$ é chamado de vetor de prioridades, pois indica a importância de cada critério para o alcance da meta proposta. Sendo $a_{i j}=\omega_{i} / \omega_{j}$, Aw resulta na equação (3), em que $M$ é a ordem (número de características) do problema.

$$
\left[\begin{array}{cccc}
\frac{\omega_{1}}{\omega_{1}} & \frac{\omega_{1}}{\omega_{2}} & \ldots & \frac{\omega_{1}}{\omega_{M}} \\
\frac{\omega_{2}}{\omega_{1}} & \frac{\omega_{2}}{\omega_{2}} & \ldots & \frac{\omega_{2}}{\omega_{M}} \\
\vdots & \vdots & \ddots & \vdots \\
\frac{\omega_{M}}{\omega_{1}} & \frac{\omega_{M}}{\omega_{2}} & \ldots & \frac{\omega_{M}}{\omega_{M}}
\end{array}\right]\left[\begin{array}{c}
\omega_{1} \\
\omega_{2} \\
\vdots \\
\omega_{M}
\end{array}\right]=\left[\begin{array}{c}
M \omega_{1} \\
M \omega_{2} \\
\vdots \\
M \omega_{M}
\end{array}\right]
$$

Com o termo b definido, pode-se agora representar o problema como

$$
\mathbf{A w}=M \mathbf{w}
$$

Note-se que as linhas $\mathbf{a}_{i}$ de $\mathbf{A}$ são linearmente dependentes — isto é, múltiplas umas das outras, de modo que

$$
\mathbf{a}_{i}=\omega_{i}\left[\begin{array}{llll}
\frac{1}{\omega_{1}} & \frac{1}{\omega_{2}} & \cdots & \frac{1}{\omega_{M}}
\end{array}\right]
$$


Sendo assim, a matriz A possui posto unitário, o que indica apenas um autovalor não nulo. Além disso, visto que os elementos da diagonal principal são unitários, pode-se concluir que

$$
\operatorname{tr}(\mathbf{A})=\sum_{m} a_{m m}=\sum_{m} \lambda_{m}=M
$$

em que o traço (soma dos elementos da diagonal principal) é igual à soma dos autovalores de $\mathbf{A}, \lambda_{m}, \mathrm{e}$, portanto, igual à ordem da matriz. Assim sendo, conclui-se que o problema $\mathbf{A w}=\mathbf{b}$ recai em um problema de autovalores e autovetores do tipo

$$
\mathbf{A w}=\lambda \mathbf{w}
$$

em que w é o autovetor de $\mathbf{A}$ associado ao maior (e único não nulo) autovalor $\lambda=M$. Essa solução é a proposta por Saaty (1990), e é denominada por Brunelli (2014) como Método do Autovetor. Saaty (1990) propõe ainda a normalização de $\mathbf{w}$ pela soma de seus elementos, de forma a obter uma composição das importâncias igual a 1 (100 \%).

Brunelli (2014) ressalta que se, na prática, os valores de $a_{i j}$ não forem obtidos diretamente dos pesos $\omega_{i}$ e $\omega_{j}$, as linhas da matriz de comparação não serão garantidamente linearmente dependentes e não existirá um vetor de pesos que sintetize perfeitamente a informação modelada na matriz $\mathbf{A}$, de forma que só é possível obter uma estimativa dos pesos e cuja consistência deve ser avaliada.

A formalização da análise de consistência é descrita por Saaty (1987), que evoca que o modelo é consistente se $\lambda_{\max }=M$, porém, devido às incertezas ocorre que $\lambda_{\max } \geq M$. Esses componentes de erro podem ser expressos nos termos conhecidos $a_{i j}$ como

$$
a_{i j}=\frac{\omega_{i}}{\omega_{j}} \epsilon_{i j} \quad, \quad \epsilon_{i j}>0
$$

de forma que

$$
\epsilon_{i j}=1+\delta_{i j} \quad, \quad \delta_{i j}>-1
$$

em que $\delta_{i j}$ é o componente de erro (perturbação) de $\omega_{i} / \omega_{j}$. Saaty (1990) postula que somente se a componente $\delta_{i j}$ for nula ocorrerá o caso $\lambda_{\max }=M$. Em outro teorema, Saaty (1990) 
estabelece que $\lambda_{\max } \geq M$ devido à inconsistência e demonstra que, se $a_{j i}=\frac{1}{a_{i j}}$ e $\mathbf{A w}=$ $\lambda_{\max } \mathbf{w}$,

$$
\lambda_{\max }-M=\frac{1}{M} \sum_{1 \leq i<j \leq M} \frac{\delta_{i j}}{1+\delta_{i j}} \geq 0
$$

A inconsistência do modelo estimado A pode ser analisada através da média negativa dos autovalores obtidos — raízes do polinômio característico de A (SAATY, 1990) — e expressa como um índice de consistência $I(\mathbf{A})$, dado por

$$
I(\mathbf{A})=\frac{\lambda_{\max }-M}{M-1}
$$

Com esse valor, obtém-se então o que é chamado de razão de consistência $-R(\mathbf{A})-$ que indica de fato o quanto a estimativa se afasta da realidade. A razão é expressa por

$$
R(\mathbf{A})=\frac{I(\mathbf{A})}{I_{M}}
$$

em que $I_{M}$ é um índice aleatório de consistência e depende do número $M$ de critérios considerados. Valores de $I_{M}$ são propostos por Saaty (1987) e podem ser observados na Tabela 2. Esses valores são obtidos pela média de 500 matrizes de ordem 1 a 10, com valores aleatórios dentro do intervalo $[1,9]$.

Tabela 2 - Índices aleatórios de consistência

\begin{tabular}{c|c|c|c|c|c|c|c|c|c|c}
\hline$M$ & 1 & 2 & 3 & 4 & 5 & 6 & 7 & 8 & 9 & 10 \\
\hline$I_{M}$ & 0 & 0 & 0,58 & 0,9 & 1,12 & 1,24 & 1,32 & 1,41 & 1,45 & 1,49 \\
\hline
\end{tabular}

Fonte: Adaptado de Saaty (1987)

Tanto Saaty (1987) quanto Saaty (1990) sugerem que valores de $R(\mathbf{A})$ até 0,1 (10\%) podem ser considerados. Para valores maiores deve ser realizada uma reavaliação dos julgamentos das importâncias relativas da matriz A, porém, observa-se nessas mesmas referências que valores superiores porém próximos são ainda utilizados. Ressalta-se também o fato de que o valor de $R(\mathbf{A})$ tende a aumentar com a quantidade de variáveis (critérios) e, portanto, requer uma avaliação qualitativa antes de qualquer descarte. 
Outra condição necessária para a consistência é a de reciprocidade, ou seja

$$
a_{i j}=\frac{1}{a_{j i}} \quad, \quad i, j=1,2, \cdots, M
$$

de forma que os elementos abaixo da diagonal principal da matriz são o inverso — recíprocos — dos valores acima da diagonal principal.

\section{Método proposto}

No modelo proposto neste trabalho, são consideradas duas categorias de trabalho principais: Ensino e Outras Atividades. As atividades que não se caracterizam como "Ensino" podem ainda se subdividir em pesquisa, atividades administrativas, dentre outras. Através do AHP é proposta uma estrutura baseada em critérios relevantes à formação da carga de trabalho desses docentes. Esses critérios servem como indicadores - cada qual com um valor de prioridade atribuído pelo modelo do AHP — para a composição da carga de trabalho e também para o diagnóstico das práticas institucionais de alocação de trabalho vigentes. Sendo assim, para a composição do segundo nível da hierarquia (vide Figura 1) são propostos os seguintes critérios:

a) Número de créditos $c$ atendidos pelo docente — Definido como

$$
c=\sum_{a=1}^{A} c_{a}
$$

em que $A$ é o número de alunos matriculados em disciplinas ministradas pelo docente e $c_{a}$ é o número de créditos contratados, vinculado ao docente, pelo $a$-ésimo aluno;

b) Número de turmas atendidas pelo docente;

c) Número de disciplinas ministradas pelo docente;

d) Número de disciplinas novas ministradas pelo docente;

e) Pontuação do docente em outras atividades - o método proposto parte do pressuposto de que uma escala de pontuação tenha sido previamente definida para as atividades que não sejam de ensino. Por exemplo, a publicação de um artigo contabiliza ao docente um determinado número de pontos, bem como um cargo de coordenação de curso de graduação ou de direção de centro. A existência desse tipo de escala é bastante comum nas instituições em razão da progressão funcional. 
Note-se que o critério 1 tem caráter diferente dos demais, uma vez que é o único que impacta diretamente na sustentabilidade financeira da instituição.

Além dos critérios 1 a 5, o modelo proposto prevê a possibilidade da consideração de um conjunto de enquadramentos administrativos que classifiquem os docentes em observação a legislação estabelecida (BRASIL, 2002). Para fins práticos, mas sem perda de generalidade, neste trabalho são assumidos enquadramentos caracterizados por funções administrativas, atividades de pesquisa e carga horária de ensino (tanto de graduação quanto de pós-graduação). Além disso, são considerados neste momento apenas enquadramentos que contemplem docentes com 40 horas semanais de dedicação à instituição:

- Administrativo 1 ou Pesquisador 1 (Adm 1/Pq 1) — docente com no mínimo 4 horasaula semanais destinadas ao ensino, distribuídas entre graduação e pós-graduação;

- Administrativo 2 ou Pesquisador 2 (Adm 2/Pq 2) — docente com no mínimo 8 horasaula semanais destinadas ao ensino, distribuídas entre graduação e pós-graduação;

- Administrativo 3 ou Pesquisador 3 (Adm 3/Pq 3) - docente com no mínimo 12 horasaula semanais destinadas ao ensino, distribuídas entre graduação e pós-graduação;

- Administrativo 4 ou Pesquisador 4 (Adm 4/Pq 4) - docente com no mínimo 16 horasaula semanais destinadas ao ensino, distribuídas entre graduação e pós-graduação;

- Docente de Tempo Integral (DTI) - docente com 20 horas-aula semanais dedicadas ao ensino de graduação;

- Docente de Tempo Parcial (DTP) - docente com 30 horas-aula semanais dedicadas ao ensino de graduação.

Os enquadramentos denominados "Adm 1/Pq 1" a "Adm 4/Pq 4" podem ser vistos como casos particulares de DTI's, cuja carga horária possui maior ênfase em atividades que não de ensino, distribuídas em administrativas e/ou de pesquisa. Esses enquadramentos também são considerados como critérios, totalizando 11 critérios $(M=11)$ no segundo nível da hierarquia. Obviamente, os enquadramentos podem ser redefinidos de acordo com as particularidades de cada instituição.

Os critérios e enquadramentos aqui propostos são, naturalmente, baseados em uma realidade institucional que, ainda que ampla e madura, é limitada. Diferentes realidades e perfis institucionais podem adequar esses critérios às suas necessidades específicas. Mais que isso, critérios distintos podem ser propostos de forma a respeitar as particularidades de cada Área do Conhecimento. Mediante eventuais adequações, entretanto, é importante que se avalie a razão de consistência, $R(\mathbf{A})$. 


\subsection{Comparação de pares}

Uma vez realizada a definição dos critérios, segue-se a metodologia de comparação de pares do AHP a fim de determinar as importâncias relativas entre essas características. Essa comparação de importâncias, inevitavelmente subjetiva, tem como base a avaliação do impacto na rotina de trabalho do docente causado pela variação de um único critério, na qual se faz a pergunta "O que seria mais trabalhoso, aumentar o critério $X$ ou o critério $Y$ ?". Os critérios são a seguir apresentados um a um sucedidos pela justificativa para a atribuição dos pesos, estes que seguem a escala definida na Tabela 1:

- Número de créditos atendidos vs. Número de turmas - Do ponto de vista de carga total de trabalho, o número de turmas sob responsabilidade de um docente é consideravelmente mais impactante (importante) que o número de créditos atendidos por ele, portanto $a_{1,2}=1 / 5$;

- Número de créditos atendidos vs. Número de disciplinas - Assumindo que o número de disciplinas ministradas pelo docente tem impacto muito maior na quantidade de trabalho que o número de créditos atendidos, atribui-se o peso $a_{1,3}=1 / 5$;

- Número de créditos atendidos vs. Número de disciplinas novas - A preparação de material e aulas para o conteúdo de uma nova disciplina a ser ministrada ocasiona talvez o maior volume de trabalho quando comparada a qualquer outra atividade docente considerada, com impacto máximo em relação ao número de créditos atendidos. Dessa forma, $a_{1,4}=1 / 9$;

- Número de créditos atendidos vs. Pontuação em outras atividades - Assume-se aqui que a produção de resultados extras em outras atividades possui impacto na carga de trabalho docente extremamente maior que um proporcional aumento do número de alunos em sala de aula. Dessa forma, $a_{1,5}=1 / 9$.

- Número de créditos atendidos vs. Enquadramentos - Assume-se que o enquadramento do docente tem sempre relevância maior ou igual (nunca menor) que o número de créditos atendidos por ele (uma vez que o enquadramento é uma decisão administrativa institucional). Por exemplo, é mais relevante para a instituição que um Próreitor em exercício execute funções de gestão que atividades de docência. Para relacionar o número de créditos atendidos com os enquadramentos, é proposta uma métrica com base no número esperado de créditos a serem atendidos por cada um dos enquadramentos, dado por

$$
n_{e}=g_{e} G+h_{e} H \quad, \quad 1 \leq e \leq 6
$$

em que $e$ referencia os seis enquadramentos definidos (critérios 6 a 11, respectivamente), $n_{e}$ é o número esperado de créditos a ser atendido por um docente do $e$-ésimo enquadramento, $g_{e}$ é a carga horária de ensino de graduação vinculada a esse enquadramento, $G$ é o número médio de alunos por turma de graduação, $h_{e}$ é a carga horária de ensino de pós-graduação vinculada ao $e$-ésimo enquadramento docente e $H$ é o número médio de alunos por turma de pós-graduação.

Para que o número de créditos $n_{e}$ referente aos diferentes enquadramentos seja 
relacionado com a escala absoluta apresentada na Tabela 1, atribui-se a maior importância relativa à categoria $\mathrm{Adm} 1 / \mathrm{Pq} 1$ (“9”), ao passo que a menor importância é imputada à categoria DTP ("1"). Dessa forma, os enquadramentos intermediários assumem valores linearmente proporcionais a essa escala. Sendo assim, o "Número de créditos atendidos" assume importância relativa mínima em comparação com o critério "Adm $1 / \mathrm{Pq} 1$ " (e, portanto, $\left.a_{1,6}=1 / 9\right)$, enquanto o "Número de créditos atendidos" assume a mesma importância relativa em comparação com o critério "DTP" $\left(a_{1,11}=\right.$ $1 / 1=1)$. Para os demais enquadramentos, são conferidos valores intermediários ajustados à escala linear descrita, com base em (15);

- Número de turmas vs. Número de disciplinas - Aumentar uma turma impacta em aumento de carga horária de ensino, enquanto aumentar uma disciplina não acarreta aumento carga horária. Note-se que o aumento de uma turma tem impacto direto na carga horária do docente (seja essa turma de uma disciplina que o docente já ministrava ou de uma disciplina nova), enquanto o aumento no número de disciplinas não impacta diretamente na carga horária, pois o docente que, por exemplo, lecionava para duas turmas de uma mesma disciplina pode agora lecionar para duas turmas de disciplinas distintas. $\mathrm{O}$ aumento do número de disciplinas, neste caso, não impacta no aumento do número de turmas e, portanto, não impacta na carga horária. Considerando-se que o docente já tenha ministrado a disciplina adicional em outro momento, assume-se que o número de turmas tem maior importância que o número de disciplinas, sendo o peso definido como $a_{2,3}=3$.

- Número de turmas vs. Número de disciplinas novas - Assume-se que ministrar uma turma a mais demanda aproximadamente o mesmo trabalho que manter-se o número de turmas sendo uma delas referente a uma nova disciplina (nunca ministrada por aquele docente, mas que seja da área de competência dele). Sendo assim, é definido o peso $a_{2,4}=1$;

- Número de turmas vs. Pontuação em outras atividades - Assume-se aqui que a produção de resultados extras em outras atividades possui impacto na carga de trabalho docente muito maior que um proporcional aumento do número de turmas. Dessa forma, $a_{2,5}=1 / 7$.

- Número de turmas vs. Enquadramentos - Esta comparação é feita com base em um ajuste linear semelhante ao realizado na comparação do número de créditos atendidos com os Enquadramentos, porém agora considera-se apenas a carga horária total de ensino de cada enquadramento (desconsiderando-se o número de alunos por turma). Dessa forma, em razão da menor carga horária de ensino, é atribuída máxima importância ao enquadramento Adm 1/Pq 1 em comparação com o número de turmas $\left(a_{2,6}=\right.$ $1 / 9)$, ao passo que na comparação com o enquadramento DTP atribui-se igual importância $\left(a_{2,11}=1 / 1=1\right)$. A comparação com os critérios intermediários resulta em valores linearmente proporcionais dentro desse intervalo conforme a escala ajustada;

- Número de disciplinas vs. Número de disciplinas novas - Do ponto de vista da carga de trabalho, assume-se que ministrar uma disciplina que já tenha sido lecionada pelo respectivo docente seja menos trabalhoso que ministrar pela primeira vez uma disciplina. Dessa forma, $a_{3,4}=1 / 7$;

- Número de disciplinas vs. Pontuação em outras atividades - Assume-se aqui que a produção de resultados extras em outras atividades possui impacto na carga de trabalho docente bastante maior que um proporcional aumento do número de disciplinas (sem aumento de carga-horária de ensino). Dessa forma, $a_{3,5}=1 / 5$. 
- Número de disciplinas vs. Enquadramentos - Nessa comparação utiliza-se a mesma metodologia da comparação "Número de turmas vs. Enquadramentos". Sendo assim, os pesos $a_{3,6}$ a $a_{3,11}$ assumem os mesmos valores de $a_{2,6}$ a $a_{2,11}$;

- Número de disciplinas novas vs. Pontuação em outras atividades - Assume-se aqui que a produção de resultados extras em outras atividades possui igual impacto na carga de trabalho docente que ministrar uma nova disciplina (sem alteração na carga horária de ensino). Dessa forma, $a_{4,5}=1$.

- Número de disciplinas novas vs. Enquadramentos - Segue-se a mesma lógica em "Número de turmas vs. Enquadramento" e "Número de disciplinas vs. Enquadramentos". Portanto $\left[\begin{array}{llll}a_{2,6} & a_{2,7} & \cdots & a_{2,11}\end{array}\right]=\left[\begin{array}{llll}a_{3,6} & a_{3,7} & \cdots & a_{3,11}\end{array}\right]=\left[\begin{array}{llll}a_{4,6} & a_{4,7} & \cdots & a_{4,11}\end{array}\right]$;

- Pontuação em outras atividades vs. Enquadramentos — É atribuída igual importância (peso 1) na comparação da pontuação em outras atividades com todos os enquadramentos, visto que cada categoria conta com uma pontuação esperada específica;

- Enquadramentos - Os enquadramentos são comparados entre si pela razão da importância de suas cargas horárias totais de ensino (descritas em "Número de turmas vs. Enquadramentos"). Assume-se que um enquadramento tem maior importância que outro na mesma proporção em que lhe cabe menor carga horária de ensino do que o outro.

Os pesos atribuídos pela análise empírica dos pares consistem nos elementos que compõem a diagonal superior da matriz de comparação, enquanto os valores da diagonal inferior são recíprocos a estes. Uma vez estabelecidos os valores necessários, é possível compor a matriz $\mathbf{A}$, conforme:

A

$$
=\left[\begin{array}{ccccccccccc}
1 & 1 / 5 & 1 / 5 & 1 / 9 & 1 / 9 & 1 / 9 & 1 / 8,35 & 1 / 7,19 & 1 / 6,04 & 1 / 3,88 & 1 \\
5 & 1 & 3 & 1 & 1 / 7 & 1 / 9 & 1 / 7,77 & 1 / 6,54 & 1 / 5,31 & 1 / 4,08 & 1 \\
5 & 1 / 3 & 1 & 1 / 7 & 1 / 5 & 1 / 9 & 1 / 7,77 & 1 / 6,54 & 1 / 5,31 & 1 / 4,08 & 1 \\
9 & 1 & 7 & 1 & 1 & 1 / 9 & 1 / 7,77 & 1 / 6,54 & 1 / 5,31 & 1 / 4,08 & 1 \\
9 & 7 & 5 & 1 & 1 & 1 & 1 & 1 & 1 & 1 & 1 \\
9 & 9 & 9 & 9 & 1 & 1 & 1,16 & 1,38 & 1,69 & 2,21 & 9 \\
8,35 & 7,77 & 7,77 & 7,77 & 1 & 1 / 1,16 & 1 & 1,19 & 1,46 & 1,91 & 7,77 \\
7,19 & 6,54 & 6,54 & 6,54 & 1 & 1 / 1,38 & 1 / 1,19 & 1 & 1,23 & 1,60 & 6,54 \\
6,04 & 5,31 & 5,31 & 5,31 & 1 & 1 / 1,69 & 1 / 1,46 & 1 / 1,23 & 1 & 1,30 & 5,31 \\
3,88 & 4,08 & 4,08 & 4,08 & 1 & 1 / 2,21 & 1 / 1,91 & 1 / 1,60 & 1 / 1,30 & 1 & 4,08 \\
1 & 1 & 1 & 1 & 1 & 1 / 9 & 1 / 7,77 & 1 / 6,54 & 1 / 5,31 & 1 / 4,08 & 1
\end{array}\right]
$$

Por fim, calculando-se o autovetor associado ao maior autovalor da matriz A, chega-se ao vetor de prioridades (w) discutido na equação (3). 


\subsection{Avaliação da carga de trabalho}

$\mathrm{Na}$ subseção anterior foi apresentada uma análise da influência dos critérios na composição da carga de trabalho de docentes, influência esta que pode ser quantificada através do vetor de prioridades (w). A análise da ocupação de um docente é agora proposta através de um vetor de avaliação, que por sua vez é ponderado pelo vetor de prioridades.

$\mathrm{O}$ vetor de avaliação do $p$-ésimo docente é definido como

$$
\mathbf{k}^{(p, e)}=\left[\begin{array}{llll}
k_{1}^{(p, e)} & k_{2}^{(p, e)} & \cdots & k_{M}^{(p, e)}
\end{array}\right]
$$

em que $e$ corresponde ao $e$-ésimo enquadramento e $M=11$ corresponde ao número de critérios. Os valores $k_{\mathbf{1}}^{(p, e)}$ a $k_{\mathbf{5}}^{(p, e)}$ correspondem a valores de referência (pré-definidos) de acordo com as características 1 a 5 da matriz de comparação ("Número de créditos $(c)$ atendidos por um docente" a "Pontuação em outras atividades") e os valores $k_{\mathbf{6}}^{(p, e)}$ a $k_{\mathbf{1 1}}^{(p, e)}$ são indicadores do enquadramento avaliado (por exemplo, se o docente pertence à categoria "Adm $1 /$ Pq 1 ", então $k_{\mathbf{6}}^{(p, e)}=\mathbf{1}$, enquanto os valores de $k_{7}^{(p, e)}$ a $k_{11}^{(p, e)}$ são nulos).

Uma vez que se deseja avaliar a influência da variação dos critérios na carga de trabalho como uma função dos enquadramentos, o vetor de avaliação deve ser normalizado (ter seus elementos divididos) por valores de referência. Por exemplo, supondo que o $p$-ésimo docente, enquadrado como "Adm 4/Pq 4", possua ocupação idêntica aos valores de referências para o respectivo enquadramento, o vetor $\mathbf{k}^{(p, 4)}$ normalizado corresponde a

$$
\overline{\mathbf{k}}^{(p, 4)}=\left[\begin{array}{lllllllllll}
1 & 1 & 1 & 0 & 1 & 0 & 0 & 0 & 1 & 0 & 0
\end{array}\right]
$$

O vetor de prioridades que pondera esse vetor de avaliação também deve ser normalizado conforme o enquadramento. O vetor de prioridades normalizado $\overline{\mathbf{w}}^{(e)}$ que pondera o vetor de avaliação do $p$-ésimo docente do $e$-ésimo enquadramento, $\overline{\mathbf{k}}^{(p, e)}$, é definido como

$$
\overline{\mathbf{w}}^{(e)}=\frac{\mathbf{w}}{\sum_{m=1}^{M} \omega_{m} k_{m}^{(p, e)}}
$$

em que $\mathbf{w}$ é o vetor de prioridades da matriz $\mathbf{A}, M=11$ é o número de critérios, $\omega_{m}$ é a $m$ ésima prioridade do vetor $\mathbf{w}$ e $k_{m}^{(p, e)}$ é o $m$-ésimo valor do vetor de características $\mathbf{k}^{(p, e)}$. Assim 
como há um vetor de características para cada enquadramento, há, portanto, um vetor de prioridades normalizado correspondente.

Como figura de mérito, propõe-se o cálculo de um valor chamado de Coeficiente de Carga de Trabalho, expresso por

$$
t^{(p, e)}=\overline{\mathbf{k}}^{(p, e)} \overline{\mathbf{w}}^{(e)}
$$

em que $t^{(p, e)}, \overline{\mathbf{k}}^{(p, e)}$ e $\overline{\mathbf{w}}^{(e)}$ são, respectivamente, o Coeficiente de Carga de Trabalho, o vetor de avaliação normalizado e o vetor de prioridades normalizado do $p$-ésimo docente do $e$-ésimo enquadramento.

\section{Simulações e resultados}

Com base na matriz A apresentada em (16) é feita a avaliação da consistência dos julgamentos, isto é, avalia-se se a análise teórica realizada na etapa de comparação de pares resulta em um modelo fidedigno. Para isto, foi calculada a razão de consistência com base nas equações (11) e (12), na qual obteve-se um valor de $R(\mathbf{A})=0,0929$. Note-se que o valor alcançado é inferior ao limiar de $10 \%$ sugerido por Saaty (1990), mesmo com o elevado número de critérios considerados, situação em que o autor inclusive cogita a necessidade de reconsideração desse limiar. Sendo assim, assume-se que a proposta de pesos que leva a (16) é consistente.

A partir desse resultado, o autovetor $\mathbf{w}$ da matriz $\mathbf{A}$ - que representa o vetor de prioridades dos critérios - foi calculado e os valores são apresentados na Tabela 3. A análise desse vetor pode ser feita em duas partes: as prioridades dos cinco primeiros critérios ("Número de créditos atendidos" até "Pontuação em outras atividades") e as prioridades dos critérios que correspondem aos enquadramentos hipotéticos propostos neste trabalho.

Para os cinco primeiros critérios, é possível observar que a maior prioridade está associada com o critério "Pontuação em outras atividades". Interpreta-se que esse critério deve ter prioridade de análise por representar maior impacto na carga de trabalho de um docente que os demais critérios. Isso se dá pelo fato de que essa pontuação está atrelada a atividades diversas, tais como tarefas administrativas (coordenação de cursos de graduação ou de centros, por exemplo) ou ainda atividades de pesquisa e/ou produção acadêmica. Essas atividades possuem valor intrínseco por vezes difícil de mensurar quando comparadas com atividades de ensino - que se relacionam diretamente com a captação de recursos financeiros para uma IES — porém são essenciais à manutenção das instituições, à atração e à retenção de estudantes. Os 
critérios "Número de disciplinas novas", "Número de turmas", "Número de disciplinas" e "Número de créditos atendidos" dispõem-se nessa ordem conforme suas prioridades, refletindo a análise feita na etapa de comparação de pares e corroborando com os julgamentos realizados. Como citado, produzir material para ministrar uma nova disciplina tem maior impacto na carga de trabalho de um docente que ministrar uma disciplina extra ou lecionar para uma turma a mais, o que também é válido se comparado o trabalho de ministrar uma disciplina para mais uma turma com o aumento do número de alunos em uma turma, por exemplo.

Tabela 3 - Vetor de prioridades $w$

\begin{tabular}{c|c|c|c}
\hline Critério & Prioridade & Critério & Prioridade \\
\hline № de créditos atendidos & 0,0147 & Adm 1/Pq 1 & 0,1994 \\
\hline № de turmas & 0,0308 & Adm 2/Pq 2 & 0,1741 \\
\hline № de disciplinas & 0,0225 & Adm 3/Pq 3 & 0,1481 \\
\hline № de disciplinas novas & 0,0506 & Adm 4/Pq 4 & 0,1222 \\
\hline $\begin{array}{c}\text { Pontuação em outras } \\
\text { atividades }\end{array}$ & 0,1123 & DTI & 0,0951 \\
\cline { 2 - 4 } & & DTP & 0,0302 \\
\hline
\end{tabular}

Fonte: Elaborado pelos autores (2020).

Quando observadas as prioridades obtidas para os critérios correspondentes aos enquadramentos, observa-se também uma confirmação dos julgamentos prévios. Esses enquadramentos necessitam ser vistos sob outro ponto de vista, isto é, suas cargas de trabalho não podem ser analisadas estritamente em função dos cinco primeiros critérios; o próprio enquadramento deve ser considerado. Isso ocorre devido ao fato de que cada enquadramento possui suas particularidades no que diz respeito à distribuição de atividades e desempenha diferentes tarefas em diferentes proporções dentro da instituição, o que foi ponderado na atribuição dos pesos de $\mathbf{A}$.

\subsection{Avaliação dos enquadramentos como critérios}

Para avaliar o método proposto e o modelo gerado através do AHP, é impreterível a definição de alguns parâmetros referentes às condições de trabalho, tanto de aspectos comuns a todos os docentes (número médio esperado de créditos atendidos em ensino de graduação e de pós-graduação) quanto de especificidades dos enquadramentos (carga horária de ensino, pontuação em outras atividades, etc). Na Tabela 4 são apresentados os valores de referência para as variáveis envolvidas no processo. Foi assumida uma média de 30 alunos por turma de graduação e uma média de 4 alunos por turma de pós-graduação (na equação (15), portanto, 
$G=30$ e $H=4)$. Tanto a carga de horária de ensino de graduação $\left(g_{e}\right)$ quanto a carga horária de ensino de pós-graduação $\left(h_{e}\right)$ são dadas em horas-aula semanais. Buscou-se através da proposição desses valores modelar um cenário possivelmente real de categorias de docentes de uma IES, respeitando as diretivas colocadas por Brasil (2002).

Tabela 4 - Valores de referência para cada enquadramento

\begin{tabular}{c|c|c|c|c|c|c} 
& \multicolumn{1}{|c|}{$\begin{array}{c}\text { Adm 1/ } \\
\text { Pq 1 }\end{array}$} & $\begin{array}{c}\text { Adm 2/ } \\
\text { Pq 2 }\end{array}$ & $\begin{array}{c}\text { Adm 3/ } \\
\text { Pq 3 }\end{array}$ & $\begin{array}{c}\text { Adm 4/ } \\
\text { Pq 4 }\end{array}$ & DTI & DTP \\
\hline No de disciplinas & 2 & 2 & 3 & 4 & 5 & 5 \\
\hline № de turmas & 2 & 2 & 3 & 4 & 4 & 8 \\
\hline Carga horária de ensino de graduação $\left(g_{e}\right)$ & 2 & 4 & 8 & 12 & 20 & 30 \\
\hline Carga horária de ensino de pós-grad. $\left(h_{e}\right)$ & 2 & 4 & 4 & 4 & - & - \\
\hline No de créditos atendidos $\left(n_{e}\right)$ & 68 & 136 & 256 & 376 & 600 & 900 \\
\hline Pontuação em outras atividades & 180 & 160 & 140 & 120 & 100 & 50 \\
\hline
\end{tabular}

Fonte: Elaborado pelos autores (2020).

Em posse desses valores, é possível determinar o vetor de avaliação descrito em (17). Uma variação dos componentes "Número de turmas", "Número de disciplinas" e "Pontuação em outras atividades" foi realizada a fim de avaliar a variação percentual do coeficiente $t^{(p, e)}$, e, para fins de comparação, foram considerados os enquadramentos “Adm 3/Pq 3" e "DTP”. O vetor de avaliação para o $p$-ésimo docente do enquadramento “Adm 3/Pq 3" pode, então, ser expresso como

$$
\overline{\mathbf{k}}^{(p, 3)}=\left[\begin{array}{lllllllllll}
\frac{256}{256} & \frac{k_{2}^{(p, 3)}}{3} & \frac{k_{3}^{(p, 3)}}{3} & 0 & \frac{k_{5}^{(p, 3)}}{140} & 0 & 0 & 1 & 0 & 0 & 0
\end{array}\right]
$$

em que o dividendo $k_{2}^{(p, 3)}$ é o "número de turmas" do $p$-ésimo docente e o divisor é o valor de referência para esse critério quando se consideram docentes da categoria "Adm 3/Pq 3", e a mesma lógica segue para o terceiro e quinto elementos do vetor, respectivamente correspondentes aos critérios "número de disciplinas" e "pontuação em outras atividades". De maneira similar, o vetor de avaliação para o $p$-ésimo docente do enquadramento "DTP" pode ser expresso por

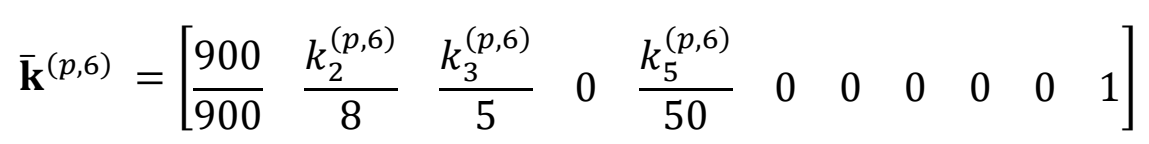


Na Figura 2 é mostrada a comparação da variação percentual do Coeficiente de Carga de Trabalho para esses dois enquadramentos de vido a uma variação no número de turmas $\left(k_{2}^{(p, e)}\right)$, mantendo-se as demais variáveis com os valores de referência (vide Tabela 4). O ponto $(0,1)$ representa os valores de referência para ambos enquadramentos, ou seja, quando um docente "Adm 3/Pq 3" ministra aulas para 3 turmas e um "DTP" ministra aulas para 8 turmas $\left(k_{2}^{(p, 3)}=3\right.$ e $\left.k_{2}^{(p, 6)}=8\right)$.

Figura 2 - Variação percentual de $t^{(p, e)}$ em função da variação do número de turmas

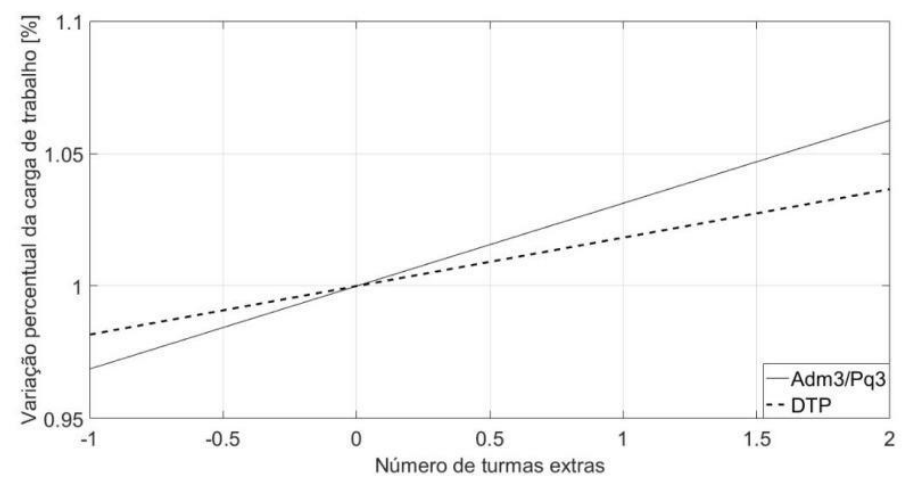

Fonte: Elaborado pelos autores (2020)

Seguindo a mesma metodologia, avaliou-se a variação percentual devido às variações de $k_{3}^{(p, e)}$ e $k_{5}^{(p, e)}$, cujos resultados podem ser vistos nas Figuras 3 e 4 , respectivamente.

Figura 3 - Variação percentual de $t^{(p, e)}$ em função da variação do número de disciplinas

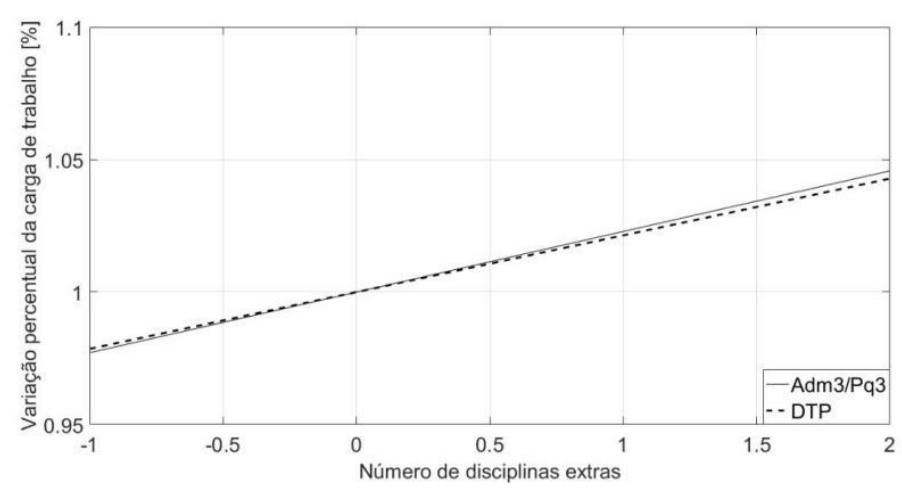

Fonte: Elaborado pelos autores (2020). 
Figura 4 - Variação percentual de $t^{(p, e)}$ em função da variação da pontuação

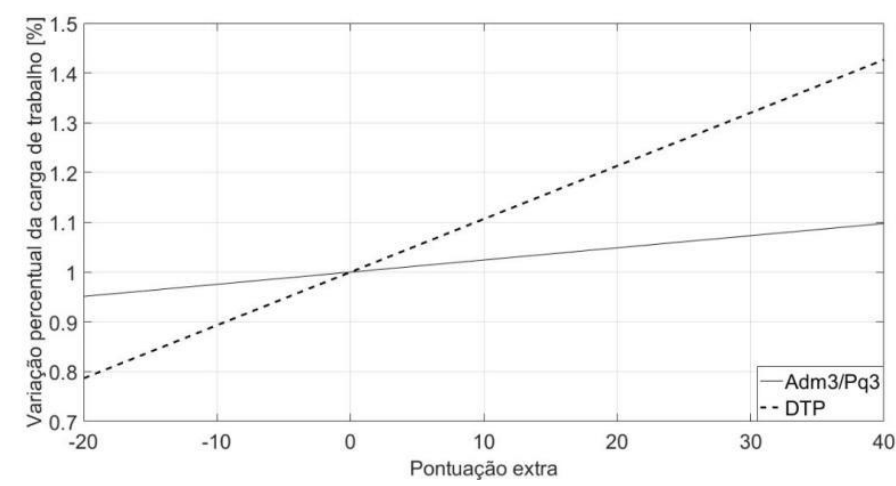

Fonte: Elaborado pelos autores (2020).

Pode-se observar pela Figura 2 que o aumento no número de turmas impacta mais a carga de trabalho de docentes do enquadramento "Adm 3/Pq 3". Atribui-se esse comportamento ao fato de que um "DTP" naturalmente já conta com uma carga de trabalho baseada majoritariamente em horas-aula, em oposição à carga de trabalho baseada em outras atividades de um "Adm 3/Pq 3". Esse comportamento se inverte se comparada a pontuação em outras atividades, como pode ser visto na Figura 4. Para um docente do enquadramento "DTP", tornase muito mais pesada a tarefa de produzir mais pontos em outras atividades do que para um “Adm 3/Pq 3", visto que sua pontuação de referência é proporcionalmente menor. Das três variáveis consideradas, a menor variação entre enquadramentos deu-se na variação do "Número de disciplinas". Interpreta-se que a tarefa de ministrar uma disciplina a mais impacta de forma igual na carga de trabalho desses enquadramentos.

Essa comparação foi estendida para todos os enquadramentos e os resultados podem ser vistos nas Figuras 5, 6 e 7. Nota-se que o comportamento observado na análise dos dois enquadramentos se replica aos demais, os quais assumem uma ordem devido aos impactos em suas distribuições de carga de trabalho, com exceção do enquadramento "Adm 2/Pq 2", cuja variação percentual de $t^{(p, 2)}$ se sobrepõe à variação de percentual $t^{(p, 1)}$ (Coeficiente de Carga de Trabalho para "Adm 1/Pq 1") em função da distribuição das atividades da carga de trabalho para esse enquadramento.

Observa-se o fato de que a variação percentual de $t^{(p, e)}$ é maior no caso da variação da pontuação, para o qual são obtidos valores superiores a $40 \%$, enquanto nos demais comparativos essa variação não excede $10 \%$, indicando que esse critério tem considerável impacto na composição da carga de trabalho dos enquadramentos. Ressalta-se também que a variação da pontuação em outras atividades mostra impacto substancialmente superior para a 
categoria "DTP" por possuir uma parcela proporcionalmente menor dessa variável em comparação com os demais enquadramentos, em termos de valores de referência.

Figura 5 - Variação percentual de $t^{(p, e)}$ em função da variação do número de turmas para todos os enquadramentos

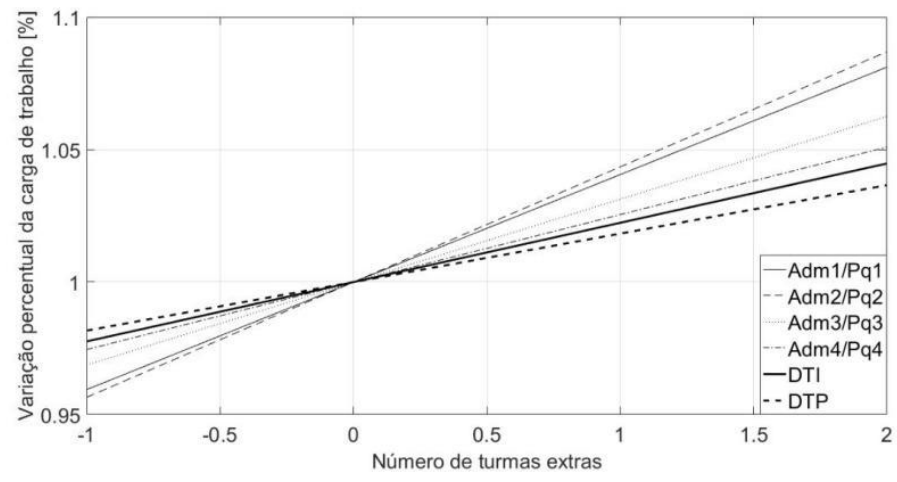

Fonte: Elaborado pelos autores (2020).

Figura 6 - Variação percentual de $t^{(p, e)}$ em função da variação do número de disciplinas para todos os enquadramentos

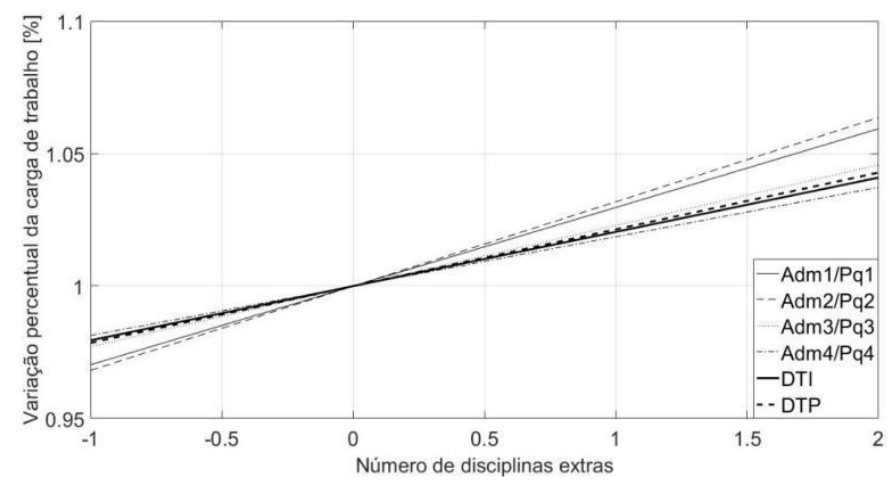

Fonte: elaborado pelos autores (2020). 


\section{Considerações finais}

A distribuição da carga de trabalho de docentes de ensino superior é um assunto complexo. Diversos fatores influenciam essa composição, sejam eles objetivos, determinados pela legislação, ou subjetivos e de difícil mensuração. Buscou-se com esse trabalho estabelecer uma métrica de avaliação para determinar como algumas das variáveis que compõem a carga de trabalho influenciam em sua composição, de forma a tornar possível a análise da qualidade das condições de trabalho dos docentes. Para isso, propôs-se o uso de uma ferramenta comum no ramo da Pesquisa Operacional, o Processo Analítico Hierárquico.

Figura 7 - Variação percentual de $t^{(p, e)}$ em função da variação da pontuação para todos os enquadramentos

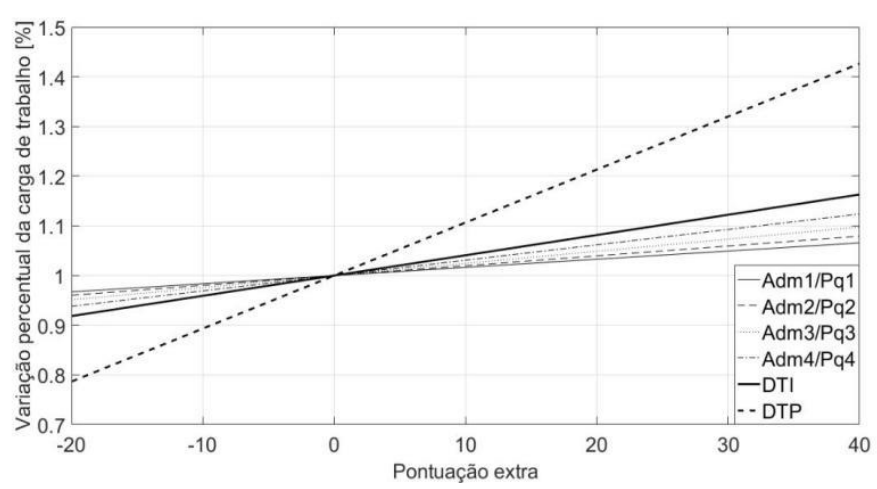

Fonte: Elaborado pelos autores (2020).

O caráter exploratório e investigativo deste trabalho abre espaço para a discussão da influência dos fatores preponderantes à formação da carga de trabalho e para novas metodologias de avaliação. Contribui-se, por fim, para os estudos em uma área que pouco avançou nos últimos 30 anos. Como principal contribuição, o trabalho propõe uma métrica (Coeficiente de Carga de Trabalho) para avaliação da ocupação docente em instituições de ensino superior. A ferramenta proposta visa a equalização das cargas de trabalho, independentemente da natureza das atividades, buscando ao final um sentimento de justiça, a harmonização das relações de trabalho e, obviamente, o consequente aumento na qualidade da produção de cada colaborador. Ainda que essa métrica possua uma base matemática consideravelmente elaborada e, portanto, talvez inacessível a profissionais de determinadas áreas do conhecimento, quando implementada computacionalmente sua utilização passa a ser simples e acessível a gestores de qualquer formação. 


\section{Referências}

BARRETT, Lucinda; BARRETT, Peter. Current practice in the allocation of academic workloads. Higher Education Quarterly, Oxford, v. 61, n. 4, p. 461-478, 2007.

BRASIL. MINISTÉRIO DA EDUCAÇÃO. SECRETARIA DE EDUCAÇÃO SUPERIOR. Manual de verificação in loco das condições institucionais: credenciamento de instituições não universitárias; autorização de cursos superiores (ensino presencial e a distância). Brasília, DF, Brasil. 2002. Disponível em http://portal.mec.gov.br/sesu/arquivos/pdf/Manual1.pdf. Acesso em: 10 jun. 2020.

BRUNELLI, Matteo. Introduction to the analytic hierarchy process. Cham: Springer, 2014.

BURGESS, Thomas F. Planning the academic's workload: different approaches to allocating work to university academics. Higher education, Netherlands, v. 32, n. 1, p. 63-75, 1996.

INEP. Instituto Nacional de Estudos e Pesquisas Educacionais Anísio Teixeira. Sinopses Estatísticas da Educação Superior - Graduação. Brasília, DF, 2018. Disponível em: http://portal.inep.gov.br/web/guest/sinopses-estatisticas-da-educacao-superior. Acesso em: 10 jun. 2020.

MU, Enrique; PEREYRA-ROJAS, Milagros. Understanding the analytic hierarchy process. In: MU, Enrique; PEREYRA-ROJAS, Milagros. Practical Decision Making, Cham, p. 7-22, 2017.

SAATY, Roseanna W. The analytic hierarchy process - what it is and how it is used. Mathematical modelling, Great Britain, v. 9, n. 3-5, p. 161-176, 1987.

SAATY, Thomas L. How to make a decision: the analytic hierarchy process. European journal of operational research, North-Holland, v. 48, n. 1, p. 9-26, 1990.

VIANA, Marcizo Veimar Cordeiro et al. O trabalho do professor na pós-graduação no Brasil após a lei n ${ }^{\circ}$ 9394/1996. Avaliação, Campinas; Sorocaba, v. 24, n. 1, p. 127-147, 2019. Disponível em: https://www.scielo.br/scielo.php?script=sci_abstract\&pid=S141440772019000100127\&lng=en\&nrm=iso\&tlng=pt. Acesso em: 12 jun. 2020. 